\title{
THE KINEMATICS OF THE IONIZED FILAMENTS IN SGR A WEST
}

\author{
E. Serabyn \\ Division of Physics, Mathematics and Astronomy \\ California Institute of Technology \\ Downs Lab of Physics, 320-47 \\ Pasadena, CA 91125
}

\begin{abstract}
The ionized gas within a few parsecs of the center of our galaxy is contained primarily in a number of thin elongated filaments (Ekers, this volume). Infrared and radio line observations have succeeded in determining the gas velocities along the majority of these filaments. A comparison of the line emission velocities with general kinematic models indicates that the motion of the filaments between 0.3 and $2 \mathrm{pc}$ from the center is consistent with predominantly orbital motion.
\end{abstract}

The kinematics of the ionized filaments within a few pc of the galactic center have been investigated by means of the [Ne II] $12.8 \mu \mathrm{m}$ fine-structure line (Serabyn $e t$ al. 1988 and references therein) and the H76 $\alpha$ recombination line (van Gorkom et al., this volume). Both lines reveal systematic velocity changes along the filaments. For example, along the western arc (for source nomenclature, see Lacy, this volume, or Serabyn et al. 1988), the velocity varies regularly from $-100 \mathrm{~km} \mathrm{~s}^{-1}$ to $100 \mathrm{~km} \mathrm{~s}^{-1}$ in moving from $35^{\prime \prime}$ South to $35^{\prime \prime}$ North of the center. (The center is taken to mean the few arc second sized region surrounding the infrared source IRS 16 and the radio point source Sgr A*). Except for effects due to clumpiness, the velocities along this filament closely match the linear rotation curve expected for gas following a circular orbit about the galactic center. This stream of gas is by now well established as the ionized inner edge of the surrounding molecular disk (see Genzel, this volume). Assuming a distance to the galactic center of $8.5 \mathrm{kpc}$, this circularly orbiting gas stream implies that a mass of $4.1 \times 10^{6} \mathrm{M}_{\odot}$ is contained within the orbital radius of $1.5 \mathrm{pc}$ (Serabyn and Lacy 1985).

The filaments which lie closer to the center also show very regular velocity variations. The velocities along the northem arm vary smoothly from 0 to $140 \mathrm{~km} \mathrm{~s}^{-1}$ along its length (van Gorkom et al. 1983, Serabyn and Lacy 1985). As to the eastern arm, the position-velocity plot of Figure 1 indicates that a single velocity component varies smoothly from 100 to $160 \mathrm{~km} \mathrm{~s}^{-1}$ along most of its length, but near the center, the velocity gradients increase markedly, and line splitting occurs (van Gorkom et al. 1983, Lacy et al. 1987, Serabyn et al. 1988). The $160 \mathrm{~km} \mathrm{~s}^{-1}$ velocity component may either drop suddenly to $0 \mathrm{~km} \mathrm{~s}^{-1}$ or increase to $300 \mathrm{~km} \mathrm{~s}^{-1}$, or these may be disconnected features. Alternatively, these two extreme velocities may be connected in a small (4") shell-like structure (Lacy $e$ t al. 1987). In any case, large spatial velocity gradients are clearly indicated in the region nearest the center.

The most difficult region to understand has been the central $10^{\prime \prime}$ region near Sgr A* and IRS 16 (sometimes called the "bar"), where several gas features overlap along the line-of-sight. The [Ne II] line has now been observed in this region with a beam as small as $1.5^{\prime \prime}$, with the result that the broad $\left(\Delta \mathbf{v}_{F W H M}>200\right.$ $\mathrm{km} \mathrm{s}^{-1}$ ), multi-component lineshapes previously observed have been clearly resolved into separate, much narrower $\left(\Delta \mathbf{v}_{\mathrm{FWHM}} \sim 50-100 \mathrm{~km} \mathrm{~s}^{-1}\right)$ features. Figure 2 shows the type of detailed information obtainable with a small beam. This figure shows a position-velocity plot of the [Ne II] emission along the filament 


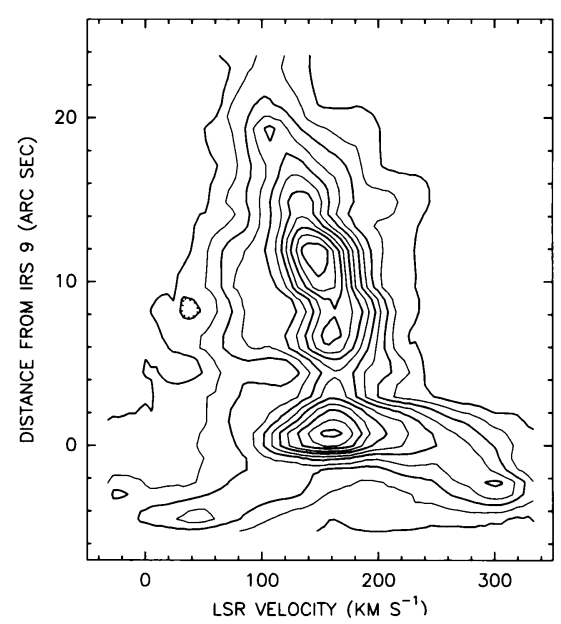

Figure 1. Position-velocity plot of the [NeII] $12.8 \mu \mathrm{m}$ emission from the eastern arm, made from the spectra of Figure 1 in Serabyn and Lacy (1988). The spectral and spatial resolutions are $25 \mathrm{~km} \mathrm{~s}^{-1}$ and 3 arc seconds, respectively. Moving upward in the figure corresponds to moving eastward along the arm.

which coincides roughly with the straight line connecting the bright infrared sources IRS 1 and IRS 2. A constant velocity gradient of $25 \mathrm{~km} \mathrm{~s}^{-1}$ arcsec${ }^{-1}$ is present for at least 7 arc seconds, and a total velocity shift of $270 \mathrm{~km} \mathrm{~s}^{-1}$ is observed. A similar position-velocity plot between the infrared sources IRS 2 and IRS 6 (west of IRS 2) also shows extended structures with similarly large velocity gradients (Figure 3).

Because these large velocity gradients have now been resolved, a much clearer picture of the velocity field in the central "bar" region has emerged. The primary consequences are that, first, several velocity components which were previously thought to belong to distinct gas structures can now be seen to be merely parts of more extended features, and second, the velocity components seen in [Ne II] can be matched one to one to bright radio continuum features. Thus, to account for most of the velocities seen in the central region where the northern, eastern and western arms come together, only 3 or 4 distinct gas features may be necessary (for details, see Serabyn et al. 1988).

The velocity vs. position information available along the filaments can be used to investigate the nature of their kinematics. As already mentioned, the gas along the western arc flows about the center in an approximately circular orbit. However, the filaments which lie nearer the center, the northern, eastern, and western arms, do not have velocity-position curves which can be interpreted so directly, and must be due to a combination of radial and azimuthal motion. Nevertheless, the nature of the dominant motion of the filaments can in fact be addressed relatively easily. Thus, for the purpose of this review, we ask simply whether the gas is predominantly orbiting about the center, flowing outward, or collapsing inward.

To facilitate the discussion, a reminder of the simplest aspects of position-velocity plots will be helpful. For gas expanding from, or collapsing directly toward, a central point, the highest velocities will be seen when looking directly toward the center. To either side of the center, the velocity drops steadily, because of projection effects. For orbital motions, the situation is exactly the opposite: independent of the exact type of orbit, be it circular or extended, somewhere near the central position the gas will be moving approximately across the line-of-sight, with $0 \mathrm{~km} \mathrm{~s}^{-1}$ radial velocity. On either side of this central zero- 


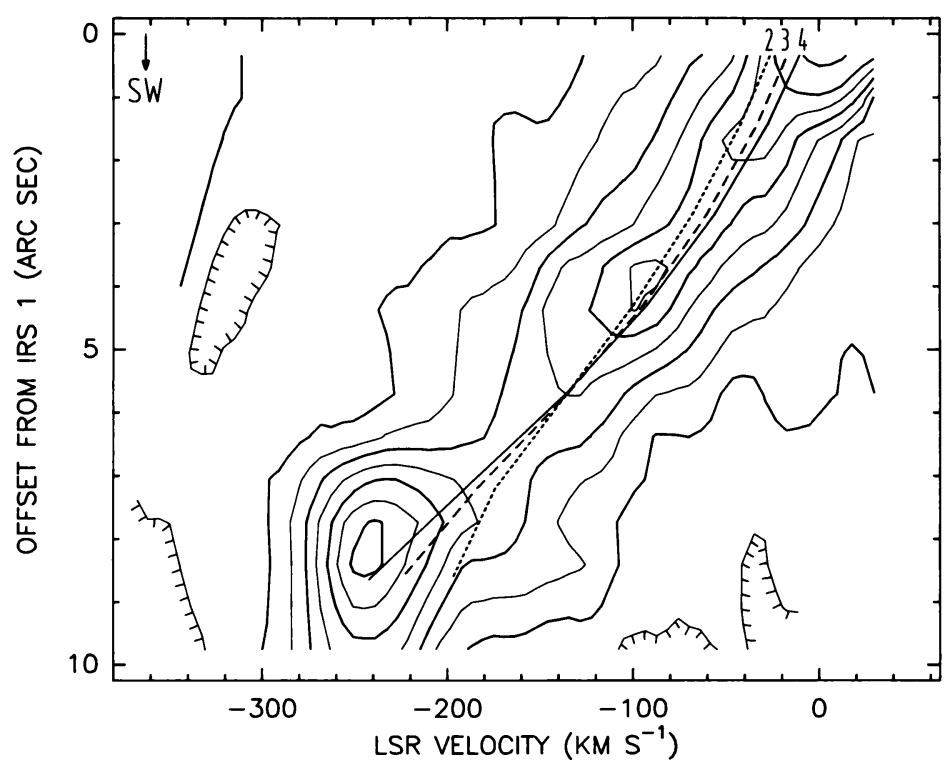

Figure 2. Position-velocity plot of the [Ne II] emission between IRS 1 (top of the figure) and IRS 2 (bottom of the figure). The data, taken from Serabyn et al. 1988, have spatial and spectral resolutions of $1.5^{\prime \prime}$ and $25 \mathrm{~km} \mathrm{~s}^{-1}$, respectively. The curves show model fits for Keplerian orbits about a point mass of 2,3 and $4 \times 10^{6} \mathrm{M}_{\odot}$.

crossing, the velocities increase in magnitude, with opposite sign on opposite sides of the center. Although for circular rotation the position-velocity plot will be linear, for general extended orbits, curvature will be present. These conclusions do not depend on the inclusion of non-gravitational forces due to, for example, magnetic fields or a drag medium, or on whether or not all of the gas is streaming along exactly the same path. Independent of these effects, a zero-crossing of the radial velocity near the central position is a signature of gas which is orbiting about the center. The exact nature of the orbital motion can of course be determined only by examining velocities along the entire gas stream.

Turning to the actual velocities along the filaments, both the western arc and the northern arm clearly show zero-crossings in radial velocity as the position of the center is crossed, and so orbital models are applicable to these two features (see e.g. Lo and Claussen 1983, Ekers et al. 1983, Schwarz 1984, Quinn and Sussman 1985, Serabyn and Lacy 1985, and Rees 1988). The eastern and western arms also show evidence of possible zero-crossings in velocity near the center, although these are more ambiguous (Figs. 1 and 3). A specific model which provides good fits to several of the gas filament locations and velocities is the Keplerian orbit model outlined in Serabyn et al. (1988). Disregarding the specifics of the model, the point to be emphasized here is that the velocities and positions along all of the extended filaments can be reproduced with orbital models. (This includes all of the velocity components in the central $15^{\prime \prime}$ region, except for the " $260 \mathrm{~km} \mathrm{~s}^{-1}$ cloud" of Lacy et al. 1980).

Models and suggestions based on radial expansion have also been put forward. These include outflowing jets (Brown 1982), acceleration by a central outflowing wind (Gatley et al. 1984, Morris and Yusef-Zadeh 1987), and ejection of magnetic loop prominences from the galactic center (Heyvaerts et al. 1988). The 


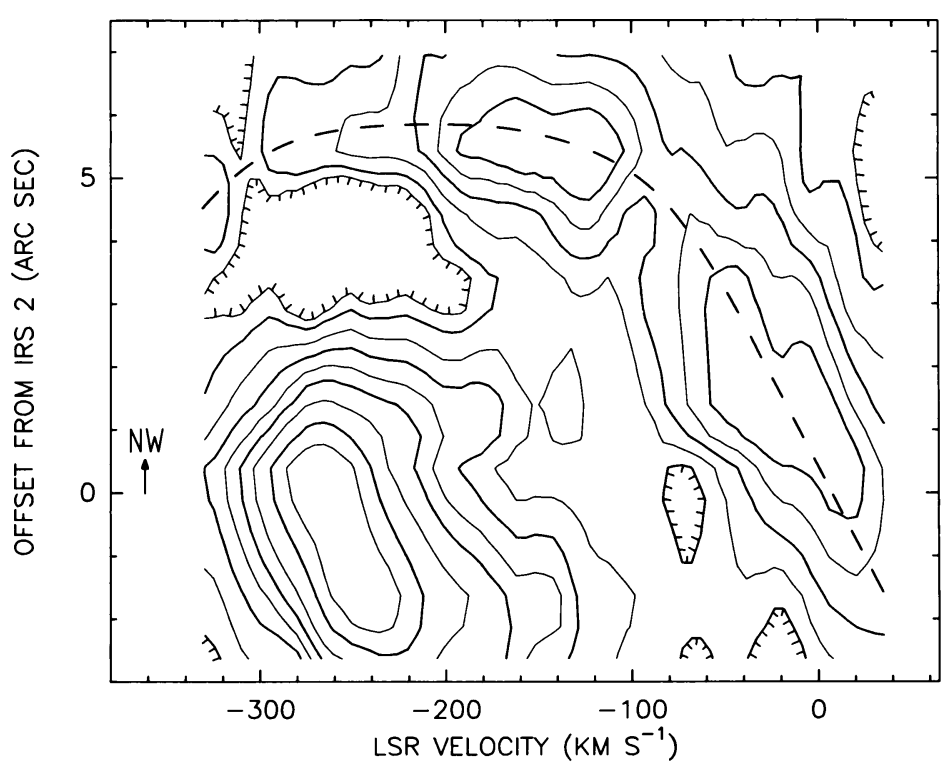

Figure 3. Position-velocity plot of the [Ne II] emission between IRS 2 (bottom of the figure), and IRS 6 (top of the figure). The observing parameters are the same as for Figure 2. The dashed line connects the features which may make up the "western arm".

first of these models predicts velocities which do not match those observed along the filaments. Although neither of the latter two models makes specific predictions for the velocities along the filaments, they can be addressed in general terms. An outflowing wind of the magnitude inferred from $\mathrm{H}$ line observations (Geballe et al. 1987) would have very significant dynamical effect on the filaments, pushing ambient gas away from the center. Thus, the position-velocity plot expected for the northern arm would be similar to that of an expanding shell. Since this is not the case, the wind's momentum would need to be scaled down significantly. The magnetic prominence model, which postulates that the "bar" is an expanding loop of gas, implies that the gas along the bar should have velocities which increase in magnitude toward the center. This is contrary to what is observed. Along the western arm, the [Ne II] velocities drop from $-160 \mathrm{~km} \mathrm{~s}^{-1}$ to $0 \mathrm{~km} \mathrm{~s}^{-1}$ as the center is approached (Fig. 3). Furthermore, the highest negative velocity gas $\left(-270 \mathrm{~km} \mathrm{~s}^{-1}\right)$ is associated not with the western arm, as previously thought, but apparently with the continuation of the northern arm past the center (Figure 2). Thus, the velocities along the bar do not fit a central expansion model. However, as the curves in Fig. 2 show, this gas is readily modeled by gas orbiting about the center.

Thus, none of the various types of expansion models can fit the measured velocity patterns along the filaments. A possible exception is the more distant section of the eastern arm, where velocities do increase toward the center. However, in this case, an explanation for the different natures of the various arms would have to be provided. (A second case in which expansion may be important is the previously suggested shell-like structure seen in the lowest part of Fig. 1. However, if confirmed with higher resolution data, this would represent a single local phenomenon, on a smaller scale than the extended filaments). For the same reason, predominantly radial infall is also ruled out. Therefore, since orbital models can fit all of the filaments, while models dominated by radial motions have difficulty, orbital models emerge as a much 
better starting point for representing the motions of the filaments. Since some radial motion is necessary, the picture of streamers of gas approaching the center (Ekers et al. 1983, Lo and Clausen 1983) continues to maintain its general applicability. (However, mathematically, the orbits can be reversed in direction and orientation, thereby also allowing the possibility of the gas orbiting outward from the center).

The question next arises of whether or not the kinematics of the filaments are dominated solely by gravitational fields, or whether other forces are also significant in determining the gas motions. Of particular interest would be the inclusion of magnetic fields in a kinematical model, to determine whether the kinks seen in the radiographs of the filaments can be reproduced (Morris and Yusef-Zadeh 1987, Killeen and Lo, this volume). Although detailed models do not yet exist for most scenarios, it is possible to make some estimates of the magnitudes of possible non-gravitational effects. Since a comprehensive discussion of these effects is presented by Lacy (this volume), they shall not be addressed here.

\section{References}

Brown, R. L. 1982, Ap. J., 262, 110.

Ekers, R. D., van Gorkom, J. H., Schwartz, U. J., and Goss, W. M. 1983, Astron. Astrophys., $122,143$.

Gatley, I., Jones, T. J., Hyland, A. R., Beattie, D. H., and Lee, T. J. 1984, MNRAS, 210, 565.

Geballe, T. R., Wade, R., Krisciunas, K., Gatley, I., and Bird, M. C. 1987, Ap. J., 320, 562.

Heyvaerts, J., Norman, C. and Pudritz, R. E. 1988, Ap. J., 330, 718.

Lacy, J. H., Lester, D. F., and Arens, J. F. 1987, in AIP conference proceedings 155, The Galactic Center, ed. D. C. Backer, p. 142.

Lacy, J. H., Townes, C. H., Geballe, T. R., and Hollenbach, D. J. 1980, Ap. J., 241, 132.

Lo, K. Y., and Claussen, M. J. 1983, Nature, 306, 647.

Quinn, P. J., and Sussman, G. J. 1985, Ap. J., 288, 377.

Rees, M. 1988, Nature, 333, 523.

Schwarz, U. J. 1984, Kapteyn Astronomical Institute Annual Report p. 48.

Serabyn, E., and Lacy, J. H. 1985, Ap. J., 293, 445.

Serabyn, E., Lacy, J. H., Townes, C. H. and Bharat, R. 1988, Ap. J., 326, 171.

Morris, M., and Yusef-Zadeh, F. 1987, in AIP conference proceedings 155, The Galactic Center, ed. D. C. Backer, p. 127.

van Gorkom, J. H., Schwarz, U. J., and Bregman, J. D. 1983, in IAU Symposium 106, The Milky Way Galaxy, ed. H. van Woerden, W. B. Burton, and R. J. Allen (Dordrecht: Reidel), p. 371. 